Revue de droit comparé du travail et de la sécurité sociale

1 | 2020

Doctrine

\title{
Le régime de la négociation collective en Grèce à nouveau bouleversé
}

Costas Papadimitriou

\section{(2) OpenEdition}

1 Journals

Édition électronique

URL : https://journals.openedition.org/rdctss/1262

DOI : $10.4000 /$ rdctss. 1262

ISSN : 2262-9815

Éditeur

Centre de droit comparé du travail et de la sécurité sociale

Édition imprimée

Date de publication : 1 avril 2020

Pagination : 188-191

ISSN : 2117-4350

Référence électronique

Costas Papadimitriou, «Le régime de la négociation collective en Grèce à nouveau bouleversé », Revue de droit comparé du travail et de la sécurité sociale [En ligne], 1 | 2020, mis en ligne le 01 novembre 2021, consulté le 11 novembre 2021. URL : http://journals.openedition.org/rdctss/1262 ; DOI : https:// doi.org/10.4000/rdctss. 1262

\section{(c) (†) $९$}

Revue de droit comparé du travail et de la sécurité sociale est mise à disposition selon les termes de la Licence Creative Commons Attribution - Pas d'Utilisation Commerciale - Pas de Modification 4.0 International. 


\section{COSTAS PAPADIMITRIOU}

Université Nationale et Kapodistriaque d'Athènes

\section{LE RÉGIME DE LA NÉGOCIATION COLLECTIVE EN GRÈCE À NOUVEAU BOULEVERSÉ}

Ledifications apportées, pendant la période de la crise économique, au droit collectif du travail grec ont été particulièrement importantes ${ }^{1}$. Après 2015 , le Gouvernement de gauche a suivi la même politique, sous la pression constante des créanciers. Ce n'est qu'en 2018 que quelques signes de changement de cap sont apparus. Certains principes traditionnels, comme le principe de faveur et le pouvoir du Ministre d'étendre l'application des conventions collectives, ont ainsi été rétablis ${ }^{2}$. En juillet 2019, le parti conservateur a gagné les élections législatives et le nouveau Gouvernement a accédé au pouvoir. Parmi ses principales préoccupations figure la législation du travail. En premier lieu, un motif sérieux ne sera plus nécessaire pour la rupture d'un contrat de travail à durée indéterminée (loi $n^{\circ} 4623 / 2019$ ). Le droit collectif du travail a été par la suite, réformé avec la récente loi $n^{\circ} 4635 / 2019$. Ces modifications du droit collectif du travail ont été présentées au motif de la nécessité de développer l'économie du pays et d'assurer le redressement de sa productivité.

Parmi ces changements, ceux qui relèvent du régime particulier des entreprises en difficulté (I), de la hiérarchie des conventions collectives (II) et du règlement des conflits collectifs (III) revêtent une importance particulière.

\section{I - LES ENTREPRISES EN DIFFICULTÉS}

La nouvelle loi $n^{\circ} 4635 / 2019$ prend en compte l'état financier des entreprises pour établir un régime particulier concernant l'application des conventions collectives. Ce régime particulier comprend trois axes : la possibilité d'exception du cadre d'application des conventions collectives (A), le concours de conventions collectives (B) et l'extension de leur application (C).

\section{A - L'EXCEPTION DU CADRE D'APPLICATION DES CONVENTIONS COLLECTIVES}

Les conventions collectives de branche et celles de métier régissent normalement les relations de travail de tous les adhérents aux organisations syndicales signataires. Jusqu'alors, la loi ne permettait pas d'exclure de leur application certaines entreprises, en tenant compte de leur situation économique.

1 C. Papadimitriou, « Le droit grec face à la crise: un passage dangereux vers une nouvelle physionomie juridique ", Revue de droit comparé du travail et de la sécurité sociale, 2012/2, p. 6.

2 C. Papadimitriou, "Les modifications récentes du droit de la négociation collective en Grèce ", Revue de droit comparé du travail et de la sécurité sociale, 2019/1, p. 218. 
La loi $n^{\circ} 4635 / 2019$ affirme que les parties signataires peuvent prévoir que ces conventions ne seront pas, que ce soit en en totalité ou en partie, appliquées aux entreprises en difficulté (régime de faillite, de pré-faillite ou de redressement financier).

\section{B - Le CONCOURS DE CONVENTIONS COLLECTIVES}

En cas de concours entre conventions collectives de branche et conventions collectives d'entreprise, c'est le principe de faveur qui est appliqué. Pourtant, dans le cas des entreprises en difficulté (régime de faillite, de pré-faillite ou de redressement financier), la nouvelle loi prévoit que c'est la convention d'entreprise, même moins favorable, qui sera appliquée. C'est ainsi qu'une entorse au principe de faveur est adoptée.

\section{C - L'EXTENSION DES CONVENTIONS COLLECTIVES}

Les conventions collectives de branche et de métier régissent normalement les relations de travail des adhérents des organisations syndicales signataires. Le Ministre du travail dispose du pouvoir d'accorder aux conventions collectives de branche et de métier une application étendue, c'est-à-dire d'imposer - lorsque les conditions prévues à cet effet sont remplies- leur application, non seulement aux adhérents des organisations syndicales signataires, mais également aux salariés non-syndiqués et aux entreprises nonmembres d'organisations patronales signataires. Cette extension d'application ne pouvait pas comprendre d'exception. Toutes les entreprises étaient concernées. La nouvelle loi prévoit que le Ministre du travail peut dorénavant, après avis du Conseil Supérieur du Travail, exclure de l'extension certaines entreprises en difficulté, que ce soit sur la totalité de la convention ou sur certaines de ces clauses.

\section{II - LA HIÉRARCHIE DES CONVENTIONS COLLECTIVES}

Le système grec de négociation collective combine tous les niveaux de conventions: les négociations se déroulent au niveau national, ainsi qu'aux niveaux de la branche, de la profession et de l'entreprise.

Au cours des dernières années de la crise économique, ce régime de hiérarchie des conventions collectives a subi une succession de modifications allant toutes dans le sens de l'abrogation du principe de faveur en cas de concours de conventions collectives, principe ancré de longue date dans le droit du travail grec. Or, au mois d'août 2018, le principe de faveur a été réintroduit en droit de travail collectif grec, ce dernier étant, de cette manière, revenu à ses origines.

En octobre 2019, le nouveau Gouvernement conservateur a introduit une exception au principe de faveur. Ainsi, en matière de concours entre une convention de branche (ou de métier) nationale et une convention de branche (ou de métier) locale, ce sont les clauses de la convention locale - mêmes si elles sont moins favorables - qui s'appliquent quand elle concourt avec une convention nationale (loi n4635/2019). Dans ce cas, le principe de spécialité est adopté. Il convient de préciser qu'aucune condition n'est prévue pour assurer la primauté de la convention locale par rapport à la convention nationale (par exemple un taux de chômage élevé ou un produit local inférieur par rapport au produit national). Il sera alors impossible de justifier une telle règle en invoquant la nécessité de développement d'une région ou en tenant compte des spécificités de son économie. Ainsi, une compétition 
vers le bas entre différentes régions du pays concernant les conditions de travail ne peut être exclue.

\section{III - LE RÈGLEMENT DES CONFLITS COLLECTIFS}

L'établissement de procédures de règlement des conflits collectifs constitue, depuis de décennies, une composante importante de relations professionnelles grecques. Cette question reste une source de débats doctrinaux, de querelles judiciaires et de tensions sociales. Même aujourd'hui, il ne semble pas que ce débat soit clos. En cas d'impasse de la négociation, une procédure de règlement est prévue par la loi n¹876/1990, et comprend la conciliation, la médiation et l'arbitrage ${ }^{3}$. C'est l'organisme de médiation et d'arbitrage, fondé par l'Etat mais géré paritairement, qui assure la médiation et l'arbitrage, tandis que la conciliation est assurée par des fonctionnaires du Ministère du travail.

L'arbitrage constitue le troisième et le dernier stade de la procédure de règlement des conflits collectifs du travail. Même si l'intervention de l'arbitre peut avoir un caractère conciliatoire, son trait caractéristique réside dans la possibilité de prononcer une sentence dont la valeur juridique ne dépend pas de l'acceptation des parties, mais qui tranche définitivement le conflit. Or, c'était la possibilité de recours unilatéral à l'arbitrage qui caractérisait le système grec. En pratique, la partie ouvrière était celle qui recourait le plus souvent tant à la médiation qu'à l'arbitrage. De ce fait, les sentences arbitrales ont été fréquemment accusées par le patronat de contribuer à l'augmentation du coût salarial et, par la suite, à la perte de productivité de l'économie grecque.

Il convient également de signaler que le Comité de la liberté syndicale de l'Organisation Internationale de Travail a affirmé qu'« il y a matière à amélioration dans l'application du principe de la négociation collective libre et volontaire et des conventions n98 et 154, ratifiées par la Grèce. Le Comité suggère donc au Gouvernement d'entreprendre des consultations avec les organisations d'employeurs et de travailleurs les plus représentatives, en vue d'envisager les mesures permettant que la possibilité de recourir à l'arbitrage obligatoire soit réservée aux services essentiels au sens strict du terme ${ }^{4}$. En revanche, la Cour de cassation grecque a affirmé que le système grec de résolution des conflits collectifs, et plus particulièrement l'arbitrage obligatoire, ne soulève aucune objection aux principes de la liberté syndicale et de l'autonomie collective ${ }^{5}$.

C'est ainsi qu'après des années de crise, le législateur grec est intervenu à plusieurs reprises (lois $n^{\circ} 3863 / 2010, n^{\circ} 3899 / 2010, n^{\circ} 4046 / 2012$ ) pour supprimer l'arbitrage "obligatoire ». C'est finalement le Conseil d'Etat grec qui a affirmé, à la suite d'un recours des syndicats de salariés et contrairement à sa jurisprudence antérieure ${ }^{6}$, que non seulement l'arbitrage " obligatoire " n'était pas contraire à l'autonomie collective et à liberté de la négociation, mais aussi que la Constitution imposait au législateur d'instaurer un système

3 C. Papadimitriou, in U. Liukkunen (ed.), " Collective Bargaining in Labour Law Regimes: A Global Perspective », Springer, 2019, p. 320.

4 Comité de la liberté syndicale, Rapport n 332, Novembre 2003, Affaire n²261 (Grèce).

5 Areios Pagos (Plen) 25/2004, DEN 2004, p. 1399. Voir aussi Conseil d'Etat 3204/1998, DEN 1999, p. 13; Conseil d'Etat 4555/1996, DEN 1997, p. 441.

6 Conseil d'Etat 3204/1998, DEN 1999, p. 13 ; Conseil d'Etat 4555/1996, DEN 1997, p. 441. 


\section{GRècE}

d'arbitrage obligatoire. Dès lors, l'organisation d'un tel système ne se présente plus selon le Conseil d'Etat comme une faculté législative, mais comme une obligation?.

Enfin, le législateur (loi $n^{\circ} 4303 / 2014$ ) obéissant à cette décision, est une nouvelle fois intervenu pour prévoir un nouveau système d'arbitrage. Ce système fonctionnait jusqu'à octobre 2019, mais d'une manière moins intense que par le passé. La loi récente n4635/2019 a restreint le champ d'application de l'arbitrage obligatoire ${ }^{8}$. Elle précise que le recours à l'arbitrage « obligatoire » ne pourra constituer que le « refuge ultime » en cas d'impasse des négociations. L'arbitrage unilatéral n'est dorénavant réservé que dans deux cas: tout d'abord dans le cas de services essentiels appartenant au secteur public, ensuite, dans le cas de différends collectifs dont le règlement est imposé par " l'intérêt général lié au fonctionnement de l'économie nationale ». La question se pose pourtant de savoir comment le système grec des relations collectives réagira, et quelles en seront les conséquences pour la paix sociale et les conditions de travail.

Pour conclure, cet ensemble de réformes pose plusieurs questions : la création d'un régime particulier de conventions collectives en matière d'entreprises en difficulté constituera-t-il une mesure susceptible de contribuer à leur rétablissement ? En outre, quelle sera l'influence de la primauté des conventions collectives locales sur les conventions nationales? Pourra-t-on découvrir des syndicats locaux disposés à signer des accords dérogatoires et dans quelles conditions? Enfin, quelle sera l'influence de l'abolition de l'arbitrage unilatéral dans les relations collectives grecques? Et peut-on imaginer un autre système fondé uniquement sur la volonté des parties? L'expérience récente prouve qu'une telle perspective est peu probable. En 2010, quand le législateur grec a aboli le recours unilatéral à l'arbitrage, le système entier est tombé en désuétude : pas de médiations, plus d'arbitrages et pas de création de systèmes conventionnels.

7 Conseil d'Etat 2307/2014, DEN 2014, p. 905

8 Certes, les parties peuvent toujours d'un accord commun initier un recours à l'arbitrage. 